Research Paper

\title{
CD73 is a hypoxia-responsive gene and promotes the Warburg effect of human gastric cancer cells dependent on its enzyme activity
}

\author{
Xiaopeng $\mathrm{Cao}^{1}$, Ziman $\mathrm{Zhu}^{2}$, Yi Cao ${ }^{3}$, Jia $\mathrm{Hu}^{4,}, \mathrm{Min} \mathrm{Min}^{1,}$ \\ 1. Department of Gastroenterology, the First Medical Center of Chinese PLA General Hospital, Beijing, China \\ 2. Department of Hepato-Pancreato-biliary Surgery, the First Medical Center of Chinese PLA General Hospital, Beijing, China \\ 3. Department of Global Health, Milken Institute School of Public Health, the George Washington University, Washington DC, USA \\ 4. Department of Oncology, the Fifth Medical Center of Chinese PLA General Hospital, Beijing, China \\ $\triangle$ Corresponding authors: Dr. Min Min. Address: Department of Gastroenterology and Hepatology, the First Medical Center of Chinese PLA General Hospital, \\ Beijing, China, Beijing, 100853, China. Telephone: (+86-10) 66947473; Fax: (+86-10) 66947473; E-mail: minmin@301hospital.com.cn. Dr. Hu Jia. Address: \\ Department of Oncology, the Fifth Medical Center of Chinese PLA General Hospital, Beijing, 100071, China. Telephone: (+86-10) 66947159; Fax: (+86-10) \\ 669474159; E-mail: hujia@301hospital.com.cn
}

(C) The author(s). This is an open access article distributed under the terms of the Creative Commons Attribution License (https://creativecommons.org/licenses/by/4.0/). See http://ivyspring.com/terms for full terms and conditions.

Received: 2021.05.06; Accepted: 2021.08.16; Published: 2021.09.03

\begin{abstract}
Background: The Warburg effect is closely associated with malignant phenotypes and poor prognosis in gastric cancer. CD73 is a glycosylphosphatidylinositol (GPI) anchored cell surface protein that functions as an oncogene in a variety of human cancers. However, the relationship between CD73 and the Warburg effect has yet to be fully understood.
\end{abstract}

Methods: Integrative analysis was performed to identify glycolysis-related genes in gastric cancer. Loss-of-function and gain-of-function are performed to demonstrate the roles of CD73 in gastric cancer cell proliferation and glycolysis. Cell biological, molecular, and biochemical approaches are used to uncover the underlying mechanism.

Results: In this study, we find that CD73 is a glycolysis-associated gene and is induced by hypoxia in gastric cancer. Genetic silencing of CD73 reduces gastric cancer cell proliferation and glycolytic ability. Opposite effects were observed by CD73 overexpression. Importantly, pharmacological inhibition of CD73 activity by APCP inhibits tumor growth, which can be largely compromised by the addition of adenosine, suggesting an enzyme activity-dependent effect of CD73 in gastric cancer. Furthermore, hijacking tumor glycolysis by 2-DG or galactose largely abrogated the oncogenic roles of CD73, indicating that CD73 promotes tumor growth in a glycolysis-dependent manner in gastric cancer. By the subcutaneous xenograft model, we confirmed the promotive roles of CD73 in regulating cell proliferation and glycolysis in gastric cancer.

Conclusions: This study provides strong evidence of the involvement of CD73 in the Warburg effect and indicates that it could be a novel antitumor strategy to target tumor metabolism in gastric cancer.

Key words: CD73; Warburg effect; Gastric cancer; NT5E; Hypoxia

\section{Introduction}

Gastric cancer is one of the most common malignant tumors in the world. The incidence and mortality of gastric cancer in East Asia and China remain high $[1,2]$. Due to the low frequency of regular gastroscopy, insidious early symptoms, poor results of radiotherapy and chemotherapy, the mortality rate of gastric cancer has remained high, the clinical outcome of patients with advanced and metastatic gastric cancer is still not optimistic [3]. Therefore, in-depth studying of the molecular mechanism involved in gastric cancer occurrence and development and developing effective diagnosis and treatment methods are urgently needed.

Due to the hypoxia and low $\mathrm{pH}$ microenviron- 
ment, the glucose metabolism of tumor cells is different from that of normal cells, which is manifested by high glycolysis but not oxidative phosphorylation and known as the Warburg effect [4, 5]. Abnormal energy metabolism especially the Warburg effect has emerged as a key feature of most tumor cells [6]. Increased Warburg effect is associated with many oncogenic phenotypes in cancers, such as rapid cell proliferation, drug resistance, and stemness [7]. Therapies targeting the Warburg effect have a profound inhibitory effect on tumor progression. In gastric cancer, glycolytic proficiency negatively affects survival outcomes of metastatic gastric cancer patients treated with paclitaxel-ramucirumab systemic therapy [8]. Notably, several reports have documented the regulators of the Warburg effect in gastric cancer, such as MACC1 [9], FBP1 [10], WTAP [11], and MUC16 [12]. However, the molecular mechanisms underlying the Warburg effect in gastric cancer remain unclear.

CD73, also known as NT5E (5'-Nucleotidase Ecto), is glycosylphosphatidylinositol-anchored cell surface protein and has been suggested to be dysregulated in most types of human cancer $[13,14]$. CD73 catalyzes the hydrolysis of AMP into adenosine and phosphate, and CD73-generated adenosine was verified to be involved in carcinogenesis, cancer apoptosis escape, and therapeutic resistance $[15,16]$. Moreover, CD73 also functions as a signal and adhesive molecule that can regulate cell interaction with extracellular matrix components, such as laminin and fibronectin, to mediate the invasive and metastatic properties of cancers. In gastric cancer, CD73 was originally found to be methylated in primary gastric cancer tissue. Subsequently, CD73 gene mutation was observed in gastric cancer metastasis [17]. Notably, CD73 overexpression in gastric cancer tissues and serums was noticed and associated with a poor clinical outcome in patients with gastric cancer [18]. Moreover, CD73 was reported to promote tumor metastasis by modulating RICS/RhoA signaling and EMT in gastric cancer [16]. However, the link between CD73 and the Warburg effect remains largely unknown.

In this study, we show that highly expressed CD73 is induced by hypoxia in gastric cancer. Genetic silencing or pharmacological inhibition of CD73 decreases the colony formation ability and glycolytic capacity of gastric cancer cells. Tumor growth advantage induced by CD73 is glycolysis-dependent and enzyme activity-dependent. Taken together, our study suggests that CD73 plays an important role in the Warburg effect by catalyzing the AMP breakdown into adenosine.

\section{Materials and methods}

\section{Gene expression analysis}

For analysis of differentially expressed genes in gastric cancer samples, data from The Cancer Genome Atlas (TCGA) and the Genotype-Tissue Expression (GTEx) were used and analyzed by the GEPIA database [19]. TCGA RNA-seq data of gastric cancer samples $(n=408)$ was used for correlation analysis and the correlation coefficient was determined by the Spearman method.

\section{Cell culture and reagents}

The eight human gastric cancer cell lines: AGS, BGC-823, HGC-27, MKN-45, and SGC-7901 were acquired from ATCC or the Institute of Biochemistry and Cell Biology, Chinese Academy of Science (Shanghai, China). These cancer cells were routinely cultured in RPMI-1640 medium or DMEM, supplemented with $10 \%$ (v/v) of fetal bovine serum (FBS, Gibco, Grand Island, NY, USA) and 1\% (v/v) streptomycin-penicillin (Sigma-Aldrich, Shanghai, China). All cells were maintained at $37^{\circ} \mathrm{C}$ in a $5 \% \mathrm{CO}_{2}$ incubator. APCP (CD73 inhibitor) and adenosine were acquired from Sigma (Shanghai, China). Galactose (S3849) and 2-Deoxy-D-glucose (2-DG, S4701) were obtained from Selleck (Shanghai, China).

\section{Cell transfection}

Short hairpin RNAs (shRNAs) against CD73 gene were transfected along with a three plasmid system (pPACKH1-REV, pPACKH1-GAG, and pVSV-G) into HEK293T cells using Lipofectamine 2000 (Invitrogen, Carlsbad, CA, USA) according to the manufacturer's protocols. The shRNA sequences were shown as follows: shRNA-1, 5'-GATCCGGAATCG TTGGATACACTTCCTTCAAGAGAGGAAGTGTAT CCAACGATTCCTTTTTTG-3'; shRNA-2, 5'-GATCCG CCGCTTTAGAGAATGCAACATTCAAGAGATGTT GCATTCTCTAAAGCGGCTTTTTTG-3'. Conditioned medium $(\mathrm{CM})$ containing retroviral particles was collected at $48 \mathrm{~h}$ after transfection, and filtered through $0.45-\mu \mathrm{m}$ filters. After incubating with conditioned medium containing retroviral particles and $5 \mu \mathrm{g} / \mathrm{ml}$ polybrene (Sigma-Aldrich, H9268, St. Louis, MO) for two days, the target cells were selected by $2 \mu \mathrm{g} / \mathrm{ml}$ puromycin for 2 weeks to yield shRNA-expressing cells. For siRNAs-mediated HIF1a knockdown experiment, gastric cancer cancer cells were seeded into 6-well plate at 70-80\% HIF1a confluency and were transfected with $15 \mu \mathrm{mol}$ siRNAs by Lipofectamine 2000 (11668019, Invitrogen, USA) according to the manufacturer's instructions. The sequences of si-HIF1a were shown as follows; si-HIF1a-1， GAGGAAGAACUAAAUCCAA; si-HIF 
1a-2, UGAUACCAACAGUAACCAA. Scramble non-target siRNAs were used as negative controls. The knockdown efficiency was detected by western blotting. For CD73 overexpression experiment, CD73 coding gene was cloned into the pcDNA3.1 expression vector. Next, the pcDNA3.1-CD73 and control vector plasmids were transfected into gastric cancer cells using Lipofectamine 2000 (11668019, Invitrogen, USA) according to the manufacturer's protocol.

\section{Real-time quantitative PCR}

Total RNA was extracted from gastric cancer cell lines with RNAiso Plus reagent (Takara, Japan) according to the manufacturers' instruction and then reverse transcribed with a PrimeScript RT-PCR kit (Takara, Japan). cDNA was amplified by PCR with 10 $\mu l$ reaction system using SYBR-Green PCR Master Mix in a Fast Real-time PCR 7500 System (Applied Biosystems, USA). All real-time qPCR reactions were completed in triplicate. The primer sequences used in this study were shown as follows: GLUT1 forward, 5'-ATTGGCTCCGGTATCGTCAAC-3', GLUT1 reverse, 5'-GCTCAGATAGGACATCCAGGGTA-3'; HK2 forward, 5'-TTGACCAGGAGATTGACATG GG-3', HK2 reverse, 5'-CAACCGCATCAGGACC TCA-3'; ENO1 forward, 5'-GCCGTGAACGAGAAGT CCTG-3', ENO1 reverse, 5'-ACGCCTGAAGAGACTC GGT-3'; PKM2 forward, 5'-AAGGGTGTGAACCTTC CTGG-3', PKM2 reverse, 5'-GCTCGACCCCAAACTT CAGA-3'; LDHA forward, 5'-ATGGCAACTCTAAAG GATCAGC-3', LDHA reverse, 5'-CCAACCCCAAC AACTGTAATCT-3'. GAPDH forward, 5'-CTGGGC TACACTGAGCACC-3', GAPDH reverse, 5'-AAGTG GTCGTTGAGGGCAATG-3'. GAPDH was served as an internal control.

\section{Western blotting}

Gastric cancer cells with indicated treatment were rinsed with cold PBS before treated with RIPA lysis buffer ( $50 \mathrm{mM}$ Tris, $0.15 \mathrm{M} \mathrm{NaCl}, 1 \mathrm{mM}$ EGTA, $1 \% \mathrm{NP} 40,0.25 \%$ SDS, $\mathrm{pH} 7.4$ ) containing protease and phosphatase inhibitors. Cell pellets were incubated for $30 \mathrm{~min}$ on ice to homogenize fully and then total proteins in the supernatant of cell lysates were collected by centrifuging at $4^{\circ} \mathrm{C}$ at $12,000 \mathrm{rpm}$ for 10 min. Total protein concentrations were measured by the BCA protein assay kit (Pierce, USA) before the experiment. Then, standard western blotting assays (SDS-PAGE) were used to analyze protein expression. The antibodies used for western blotting analysis in this study included: anti-CD73 (Abcam, ab133582, 1:1,000 dilution), anti-HIF1a antibodies (Abcam, ab51608, 1:1,000 dilution), and anti- $\beta$-actin antibody (Sigma, A2228, 1:5,000 dilution). After incubation with corresponding species-specific secondary HRP-conjugated antibodies, the target protein bands were visualized by an ECL imaging system.

\section{Glucose uptake and lactate production}

Glucose and lactate levels in the culture medium were detected using Glucose Assay kit (Biovision, Milpitas, CA, USA) and Lactate Assay kit (Biovision, Milpitas, CA, USA), respectively. In brief, gastric cancer cells were seeded at $5 \times 10^{5}$ cells in 6 -cm cell culture dishes and allowed to grow for $24 \mathrm{~h}$ with indicated treatment. After $24 \mathrm{~h}$, the culture medium was collected and centrifuged at 2,000 rpm for $5 \mathrm{~min}$ to obtain the supernatant without cell debris. Finally, glucose and lactate assays were performed according to the manufacturer's instructions, and colorimetric density was assessed using a Multifunctional microplate reader.

\section{Plate colony formation assay}

Gastric cancer cells were resuspended and plated in 6-well plates at a density of 500 cells per well and allowed to grow for 10-14 days with indicated treatment. Then the cells were fixed with $4 \%$ paraformaldehyde for $15 \mathrm{~min}$ at room temperature and stained with $0.5 \%$ crystal violet for $15 \mathrm{~min}$. The experiment was performed in triplicate.

\section{Chromatin immunoprecipitation-PCR}

Chromatin Immunoprecipitation (ChIP) experiment was performed in gastric cancer cells under $20 \%$ $\mathrm{O}_{2}$ and $1 \% \mathrm{O}_{2}$ conditions. In brief, gastric cancer cells with indicated treatment were fixed with $1 \%$ formaldehyde for $10 \mathrm{~min}$ at room temperature followed by incubation with $0.125 \mathrm{M}$ glycine for 10 min. Next, cells were collected, washed with ice-cold PBS three times, resuspended in lysis buffer, supplemented with protease inhibitor cocktails, and sonicated to obtain chromatin fragments of about 500-1,500 bp. The supernatants were obtained and incubated with anti-HIF1a antibody (F7425) and Dynabeads Protein $\mathrm{G}$ at $4^{\circ} \mathrm{C}$ overnight. The beads were washed, and the precipitated chromatin complexes were collected, purified, and de-crosslinked. Finally, the precipitated DNA fragments were analyzed by RT-PCR.

\section{Animal studies}

Male BALB/c athymic nude mice (5-6 weeks old and weighing 18-20 g) were purchased from the Jiesijie Animal Center (Shanghai, China). To establish the gastric cancer xenograft model, $2 \times 10^{6}$ stable CD73-knockdown and sh-Ctrl gastric cancer cells were suspended in $100 \mu \mathrm{l}$ PBS and inoculated subcutaneously into the flanks of nude mice. After 4 weeks, the mice were sacrificed, and the tumors were 
excised and subjected for further analysis. These experiments were performed in full accordance with the recommendations in the Guide for the Care and Use of Laboratory Animals of the National Institutes of Health, and all the animal experiments were approved by the Animal Experimental Ethics Committee.

\section{Immunohistochemistry}

Standard procedure of Immunohistochemistry (IHC) analysis was performed. In brief, tissue sections $(4 \mu \mathrm{m})$ were firstly deparaffinized, followed by citrate-based antigen retrieval and blocking with $0.2 \%$ endogenous peroxidase. After blocking with 10\% bovine serum albumin (BSA, Sangon, Shanghai, China) for $45 \mathrm{~min}$ at room temperature, the slides were incubated with primary antibodies at $4{ }^{\circ} \mathrm{C}$ overnight. For Ki67 staining, sections from xenograft tumor tissues were incubated with a monoclonal mouse anti-Ki67 antibody (Cell Signaling Technology; 1:8,000 dilution; \#2586), followed by staining with an HRP-conjugated secondary antibody (Abcam; 1:300 dilution; ab6728). Immunoreactivity was generated by the DAB system. Finally, sections were counterstained by hematoxylin, and images were analyzed with a NIKON microscope.

\section{Statistical analysis}

Data were represented as mean \pm standard deviation (SD). GraphPad 6.0 (GraphPad Software Inc., San Diego, CA) was used for statistical analysis. The correlation of gene expression was evaluated by Spearman's correlation. P-values were calculated by two-tailed unpaired Student's t-test or one-way ANOVA by two-tailed post hoc Dunnett's multiple comparison test. $\mathrm{P}<0.05$ was considered to be statistically significant.

\section{Results}

\section{Identification of glycolysis-associated genes in gastric cancer}

To investigate the differentially expressed genes (DEGs) related to gastric cancer cell glycolysis, the expression profiles of mRNA data of stomach adenocarcinoma (STAD) were acquired from The Cancer Genome Atlas (TCGA) and used for subsequent analysis. Based on a glycolysis gene signature reported previously [20], the STAD cohort was divided into two groups: glycolysis-low $(n=204)$ and glycolysis-high $(n=204)$. Gene set enrichment analysis (GSEA) showed that HALLMARK glycolysis gene signature was significantly enriched in the glycolysis-high samples (Figure 1A). The heatmap of differentially expressed genes between glycolysishigh and glycolysis-low groups was shown in Figure
1B. Among the identified DEGs, we found that 7 genes (IGFBP7, LOX, NRP1, PRSS3, DPP3, EFNA3, and $C D 73)$ were also differentially expressed in tumor tissues and associated with a poor or better prognosis in patients with gastric cancer (Figure 1C). Among these genes, no reports regarding the role of $C D 73$ in reprogrammed metabolism were found. Therefore, CD73 was of particular interest to investigate.

\section{CD73 is induced by hypoxia in gastric cancer}

By coexpression analysis, we found a close correlation between CD73 and hypoxia gene signatures (Figure 2A). By western blotting analysis of CD73 in gastric cancer cell lines, we observed that CD73 was highly expressed in gastric cancer cells compared with the nonmalignant GES1 cells (Figure 2B). To determine whether CD73 is induced by hypoxia in gastric cancer, we cultured BGC-823 cells under both normoxic and hypoxic conditions (Figure 2C). As a result, CD73 expression was significantly boosted by hypoxia at protein levels (Figure 2C), indicating a regulatory role of HIF1a in CD73 expression. Similarly, a chemical inducer of HIF1a, $\mathrm{CoCl}_{2}$, phenocopied the effect induced by hypoxia (Figure 2D). As the next line of evidence, genetic silencing of HIF1a largely attenuated hypoxiainduced CD73 expression (Figure 2E). Moreover, chromatin immunoprecipitation data showed that HIF1a interacted directly with CD73 gene promoters under hypoxia (Figure 2F). Therefore, highly expressed CD73 may be induced by hypoxia in gastric cancer.

\section{CD73 regulates the Warburg effect in gastric cancer}

To demonstrate whether CD73 plays a role in gastric cancer glycolysis, in vitro loss-of-function studies were first carried out. Two shRNAs against CD73 led to a $>70 \%$ reduction of CD73 protein expression in both HGC-27 and SGC-7901 cells (Figure 3A). Consistent with the knockdown efficiency, reduction in CD73 expression led to a significant decrease in glucose uptake (Figure 3B), lactate production (Figure 3C), and expression of glycolytic genes (Figure 3D) in HGC-27 and SGC-7901 cells. To further confirm the potential regulatory role of CD73 in gastric cancer glycolysis, we overexpressed CD73 in BGC-823 cells (Figure 3E). As expected, ectopic expression of CD73 enhanced the Warburg effect as evidenced by glucose uptake, lactate release, and expression of glycolytic genes (Figure 3F). To further investigate whether the promotive effect of CD73 on the cell glycolysis was dependent on its enzyme activity or was mainly the results of its non-enzymatic function, we used $50 \mu \mathrm{M}$ 
APCP (the specific inhibitor of CD73 enzymatic activity) to block the CD73 enzyme activity. The results showed that APCP treatment inhibited cell glycolysis, which can be largely restored by the addition of adenosine (Figure $3 \mathrm{G}$ and $\mathbf{3 H}$ ), indicating that CD73 regulated the Warburg effect via enzyme-dependent activity.

\section{The growth advantage induced by CD73 is glycolysis-dependent in gastric cancer}

Next, we studied the potential oncogenic roles of CD73 in gastric cancer. By plate colony formation assay, we found that CD73 knockdown contributed to decreased proliferative capabilities in HGC-27 and SGC-7901 cells (Figure 4A). Because that CD73 can promote the glycolytic ability of gastric cancer cells, we next tested whether CD73 affects tumor growth via modulation of tumor glycolysis. To address this hypothesis, we evaluated the effect of CD73 overexpression on gastric cancer cell proliferation. CD73 overexpression led to a significant increase in cell proliferation of BGC-823 cells, which can be largely abrogated by the addition of the known glycolysis inhibitor 2-DG (Figure 4B).Next, we grew BGC-823 cells in the culture medium containing galactose instead of glucose. The rate at which galactose enters glycolysis is much lower than that of glucose. Notably, galactose largely compromised CD73-mediated upregulation of cell proliferation (Figure 4C). Moreover, HGC27 cells were pretreated with glycolysis inhibitor 2-DG, followed by combined treatment with $50 \mu \mathrm{M}$ APCP. As shown in the Figure 4D, 2-DG and APCP can suppress HGC27 cell proliferation, respectively. However, the tumor-
A

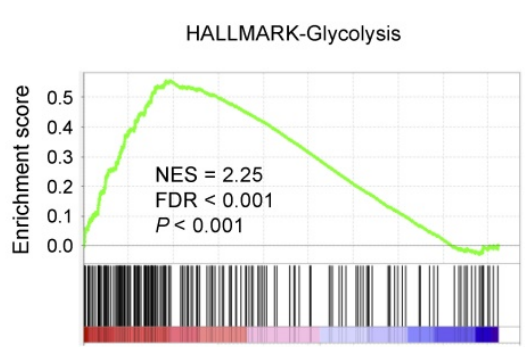

High group

C

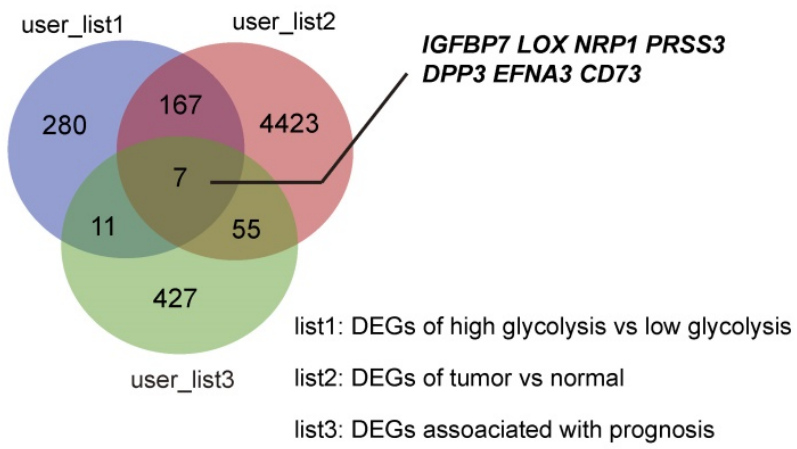

B

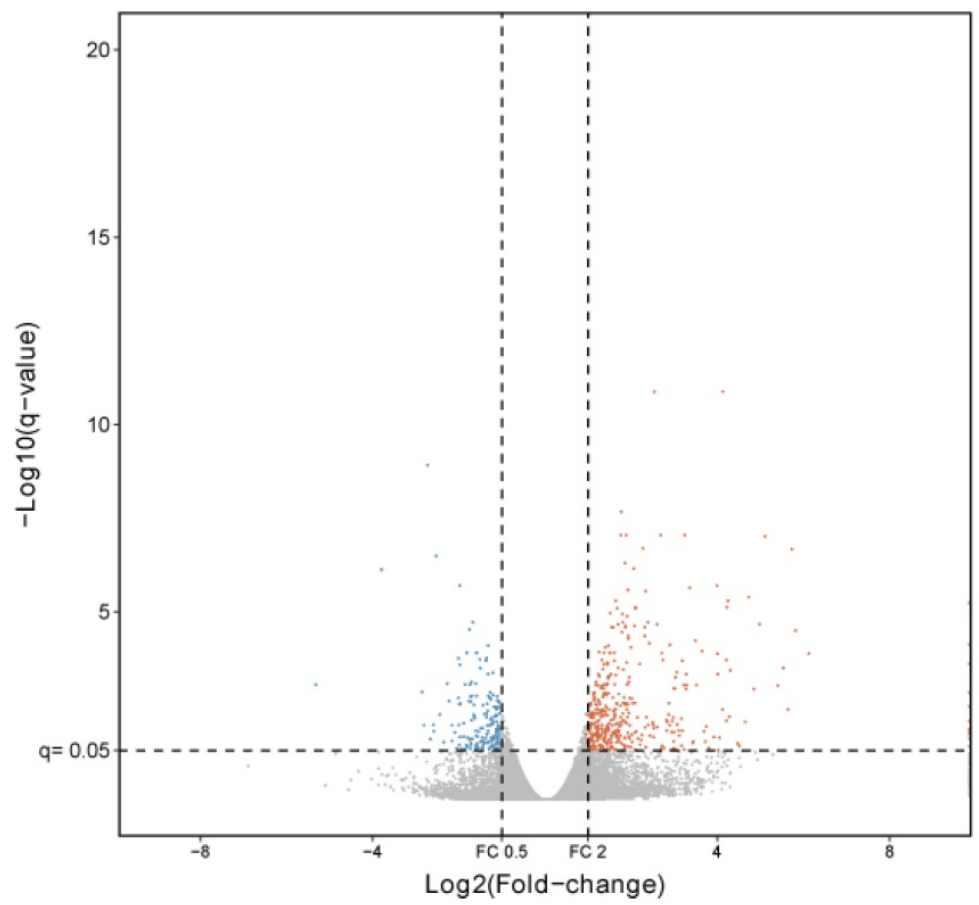

Down regulation

Not Significant

Up regulation

Figure 1. Identification of glycolysis-associated genes in gastric cancer. (A) Gene set enrichment analysis (GSEA) on Hallmark glycolysis gene sets across the glycolysis-low and glycolysis-high samples. NES, normalized enrichment score (NES); false discovery rate (FDR) was set at 0.25 . (B) Volcano plot of differentially expressed genes (DEGs) between gastric cancer samples clustered in glycolysis-low and glycolysis-high groups. (C) Venn diagram showed DEGs related to glycolysis, DEGs between tumor and normal tissues, and genes associated with a better or a poor prognosis in gastric cancer. 
suppressive effect was not further enhanced by combined treatment. Collectively, these data above suggest that CD73 couples cell glycolysis to tumor cell proliferation in gastric cancer.

\section{CD73 knockdown suppresses tumor growth in vivo}

To test the in vivo effect of CD73 knockdown on tumor growth, a subcutaneous xenograft model was

A

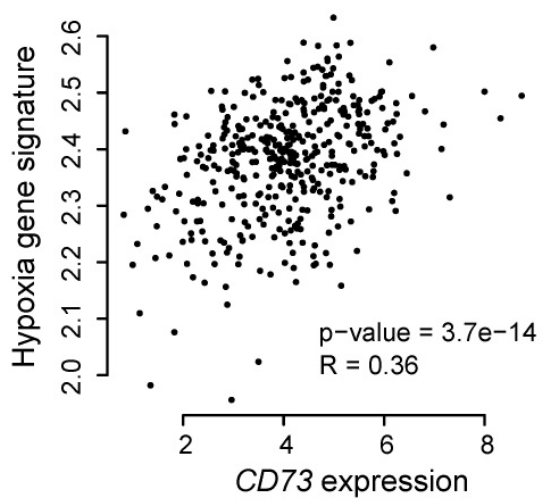

C

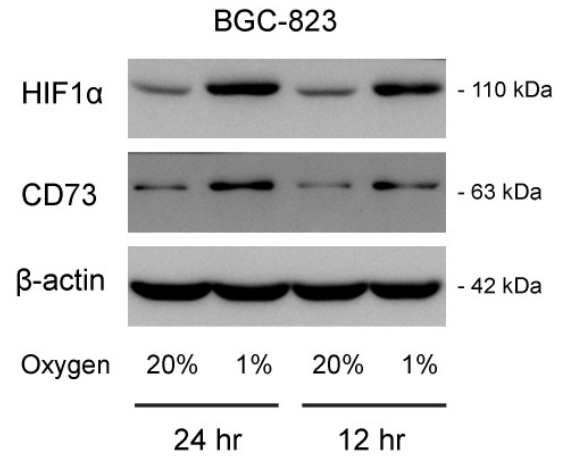

E

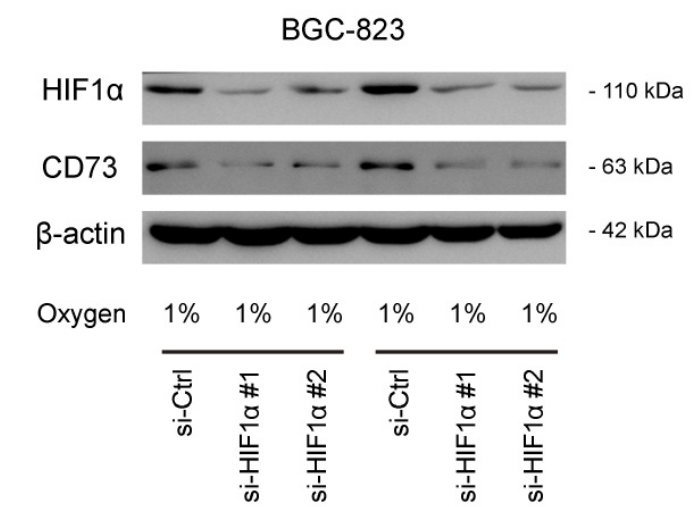

generated. As a result, stably knockdown of CD73 significantly retarded tumor burden as evidenced by tumor weight, tumor volume, and the proliferation index Ki67 (Figure 5A-C). By real-time qPCR analysis, we found that CD73 knockdown can lead to significant down-regulation of glycolytic genes (GLUT1, HK2, ENO1, and LDHA) in tumor tissues from the subcutaneous xenograft model (Fig. 5D).

B

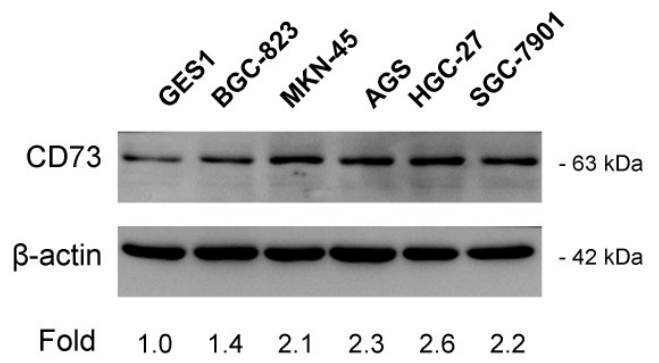

D

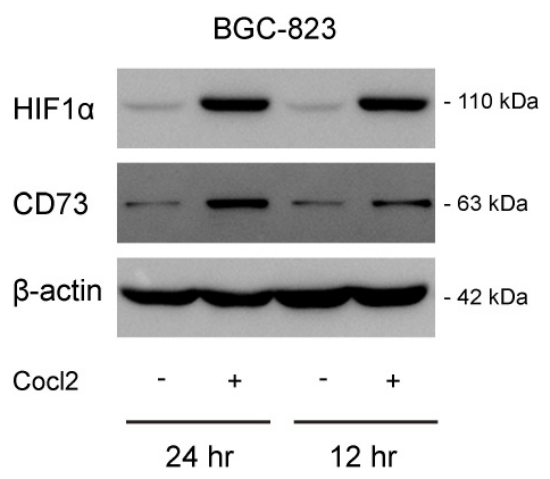

$\mathbf{F}$

BGC-823

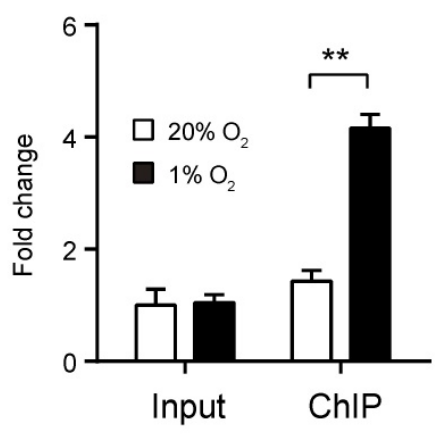

Figure 2. CD73 is induced by hypoxia in gastric cancer. (A) Correlation analysis of CD73 expression and hypoxia gene signature in gastric cancer; data were obtained from TCGA cohort. (B) Western blotting analysis of CD73 expression in gastric cancer cell lines and GES1 cell line. (C) BGC-823 cells were cultured under hypoxia ( $1 \%$ O $\left.\mathrm{O}_{2}\right)$ and normoxia $\left(20 \% \mathrm{O}_{2}\right)$ for 12 or $24 \mathrm{~h}$, followed by detection of $\mathrm{CD} 73$ expression with western blotting. (D) BGC-823 cells were cultured with the treatment of $100 \mu \mathrm{M} \mathrm{CoCl} 2$ or not for 12 or $24 \mathrm{~h}$, followed by detection of CD73 expression with western blotting. (E) Under hypoxic culture condition (1\% $\mathrm{O}_{2}$ ), CD73 expression in BGC-823 cells in the presence or absence of HIFla knockdown was analyzed by western blotting. (F) Chromatin immunoprecipitation-PCR validation of the regulatory role of HIF1a in CD73 expression. Input and ChIP cycle threshold $(\mathrm{Ct})$ values were normalized separately to control as 1 . $* * P<0.01$. 
A

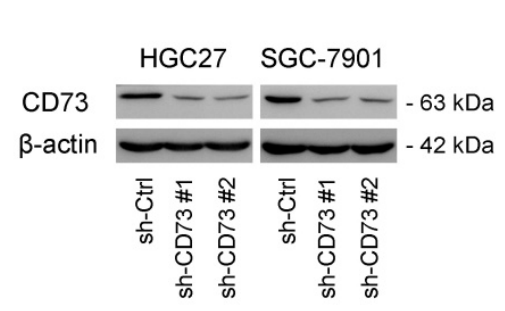

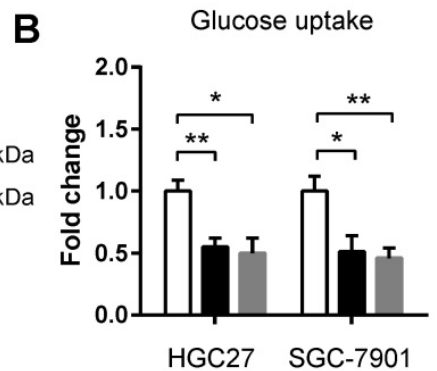

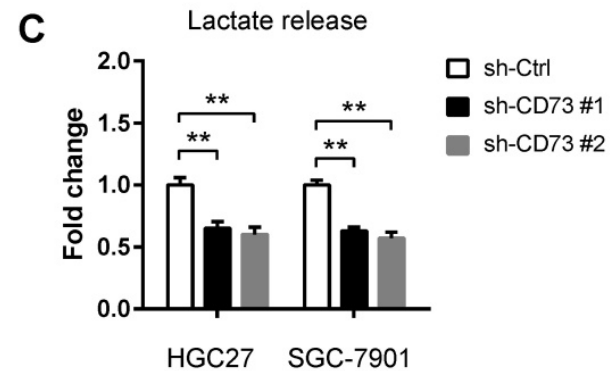

D

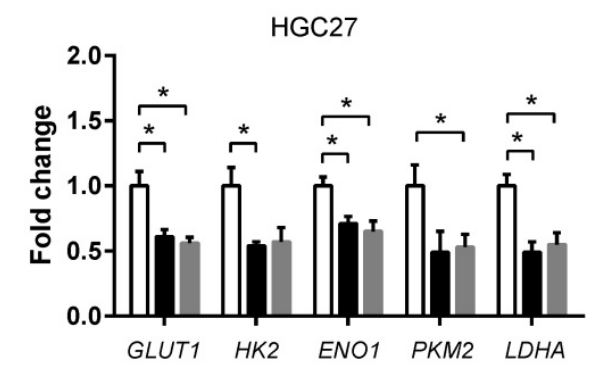

E

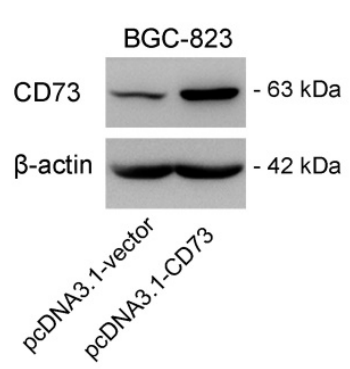

G

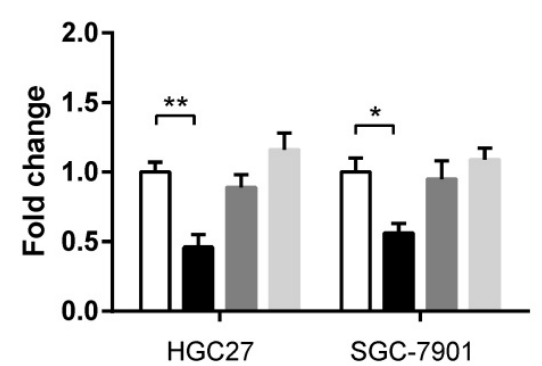

$\mathbf{F}$

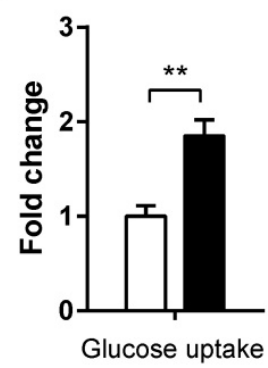

H

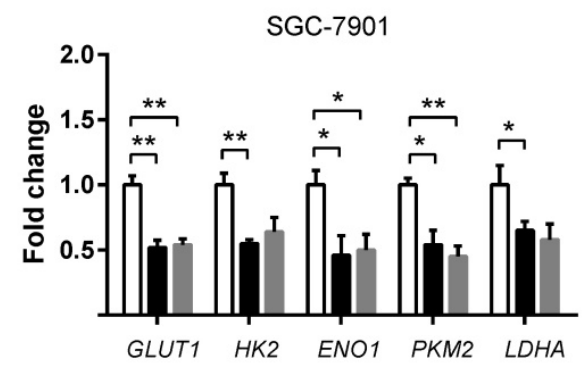

$\square$ pcDNA3.1-vector

pcDNA3.1-CD73
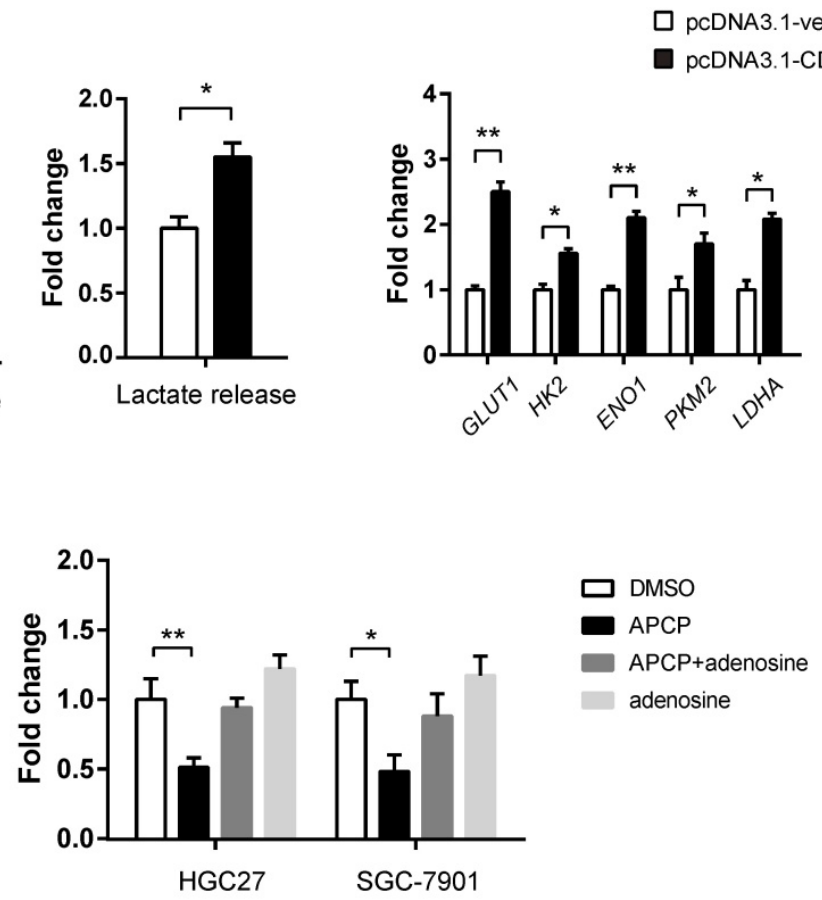

Figure 3. CD73 regulates the Warburg effect in gastric cancer. (A) The knockdown efficiency of two independent shRNAs against CD73 in HGC-27 and SGC-7901 cells was analyzed by western blotting analysis. (B-D) Effect of CD73 knockdown on the glucose uptake $(B, n=3)$, lactate release $(C, n=3)$, and expression of glycolytic genes $(D, n=3)$ in HGC-27 and SGC-7901 cells. (E) The overexpression efficiency of CD73 in BGC-823 cells was confirmed by western blotting analysis. (F) Effect of CD73 overexpression on the glucose uptake $(n=3)$, lactate release $(n=3)$, and expression of glycolytic genes $(n=3)$ in BGC-823 cells. $(\mathbf{G})$ The effect of APCP $(50 \mu M)$ and adenosine $(10 \mu \mathrm{M})$ on the glucose uptake of HGC-27 and SGC-7901 cells. $(\mathbf{H})$ The effect of APCP $(50 \mu \mathrm{M})$ and adenosine $(10 \mu \mathrm{M})$ on the lactate release of HGC-27 and SGC-7901 cells. $*_{p}<0.05 ; * *_{p}<0.01$.

\section{Discussion}

CD73 plays diverse role in human cancers. In this study, we investigated (i) the glycolysis-associated genes in gastric cancer, (ii) the effect of CD73 on tumorigenic potential and the Warburg effect, (iii) whether increased growth advantage induced by CD73 is glycolysis-dependent, and (iv) whether CD73-mediated glycolysis is dependent on its enzyme activity. Our results suggest that CD73 is profoundly implicated in the Warburg effect and can be exploited as a potential target for gastric cancer therapy.

Aberrant expression of CD73 has been reported in many cancers, such as lung cancer, colorectal cancer, breast cancer, prostate cancer, ovarian cancer, pancreatic cancer, and gastric cancer [21-26]. However, little is known about the reason for CD73 
dysregulation. Previously, CD73 is identified as a target of miR-30a-5p and plays an important role in the pathogenesis of non-small cell lung cancer [27]. Moreover, accumulated evidence suggests the link between CD73 and hypoxia. For instance, high CD73 expression in lung cancer cells is directly linked with hypoxia-inducible factor HIF1a expression by cancer cells [28]; in fulminant acute liver failure [29], CD73 expression can be increased by HIF1a; in gastric cancer, CD73 expression correlates closely with HIF-1a expression [18]. Consistent with these observations, we provided proof of principle data of hypoxia-dependent expression of CD73 in gastric cancer. Moreover, our findings further broaden the roles of CD73 in gastric cancer and suggest a glycolysis-dependent effect of CD73. Targeting CD73 by genetic inhibition or monoclonal antibodies might be a preferred way. In gastric cancer, inhibition of CD73 in primary tumors could dramatically abolish the effects of CD73 and eventually suppress experimental metastasis in both peritoneal seeding and hematogenous metastasis model [16]. Moreover, recent studies have revealed an enhanced antitumor effect of anti-PD-1 or anti-CTLA-4 mAb in a mouse model when combined with anti-CD73 mAb [30, 31]. Therefore, our and other studies support the possibility of targeting CD73 from diverse perspectives.
A HGC27
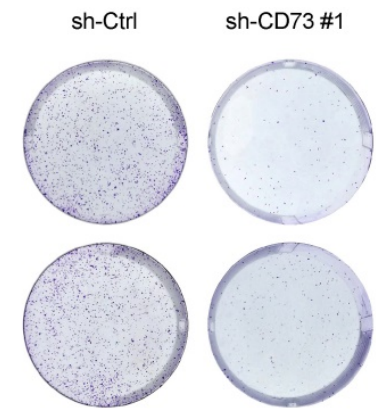

B

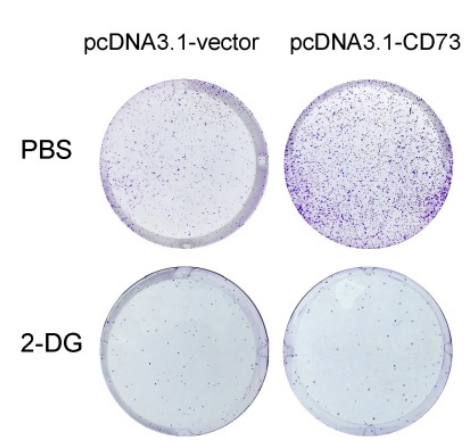

D

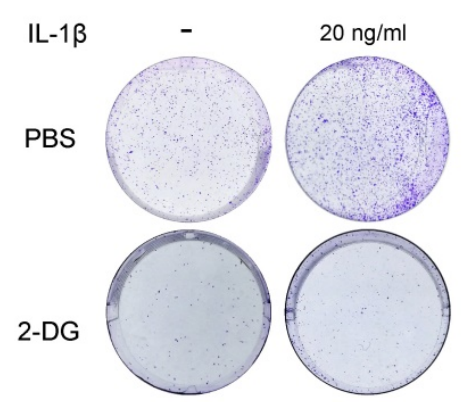

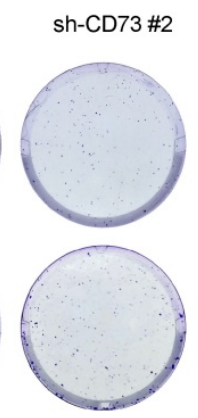

$\square$ pcDNA3.1-vector pcDNA3.1-CD73

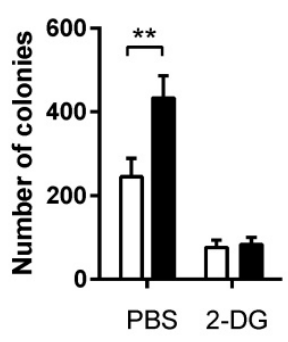

PBS 2-DG

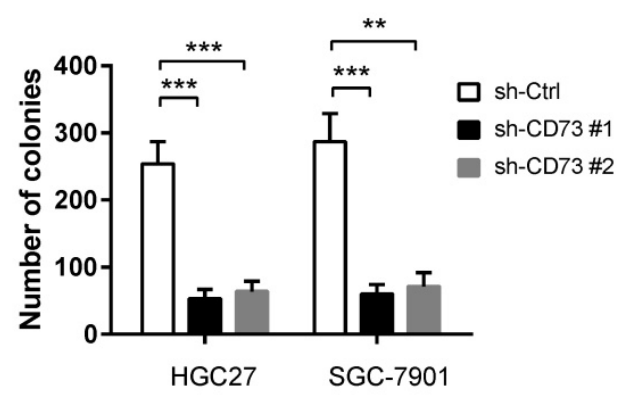

C

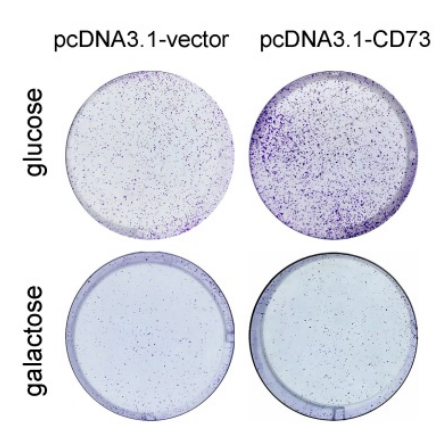

$\square$ pcDNA3.1-vector
$\square$ pcDNA3.1-CD73

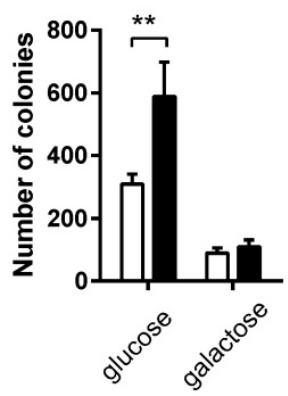

E

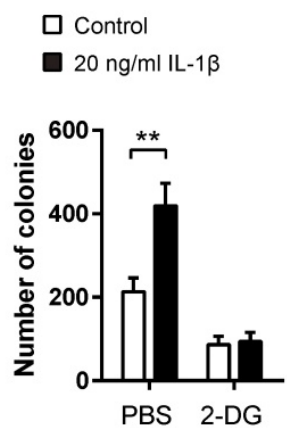

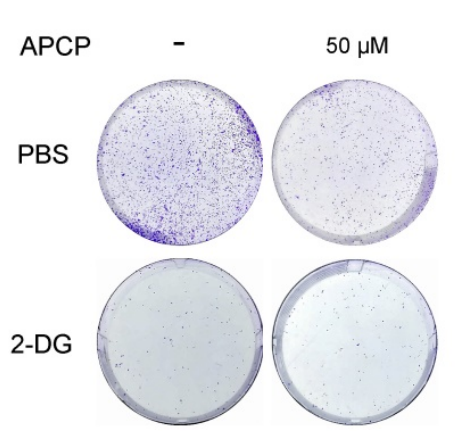

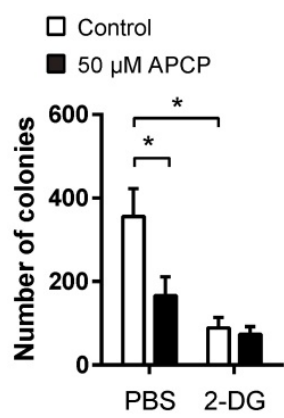

Figure 4. The growth advantage induced by CD73 is glycolysis-dependent in gastric cancer. (A) The effect of CD73 knockdown on in vitro proliferation of HGC-27 and SGC-7901 cells was analyzed by colony formation assay $(n=3)$. (B) The effect of CD73 overexpression on the proliferation of BGC-823 cells in the presence or absence of $50 \mathrm{mM}$ 2-DG was analyzed by plate colony formation assay. (C) BGC-823 cells were cultured in media containing 25 mM galactose but no glucose and plate colony formation experiment was performed. (D) The effect of 2-DG on the proliferation of HGC27 cells in the presence or absence of $50 \mu M$ APCP was analyzed by plate colony formation assay. $* *_{p}<0.01 ; * * * p<0.001$. 
A

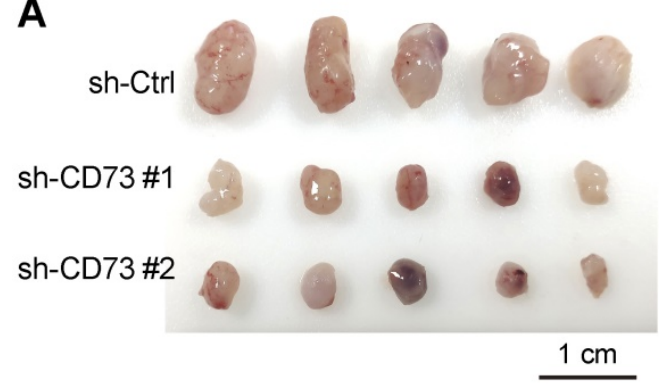

C

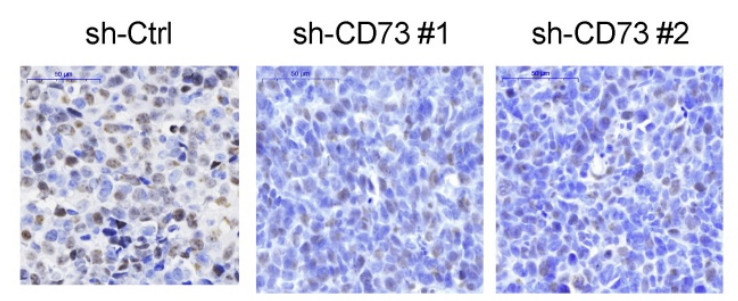

B

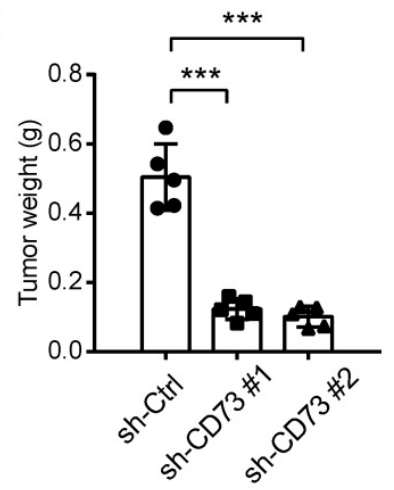

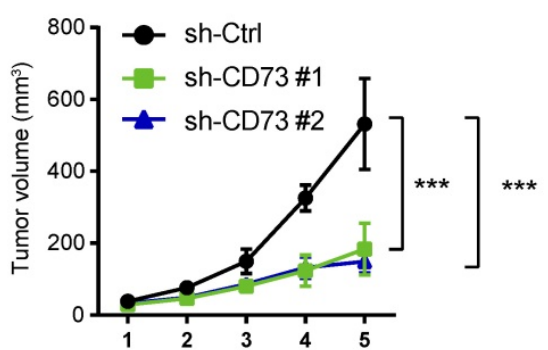

sh-Ctrl

sh-CD73 \#1

sh-CD73 \#2

D

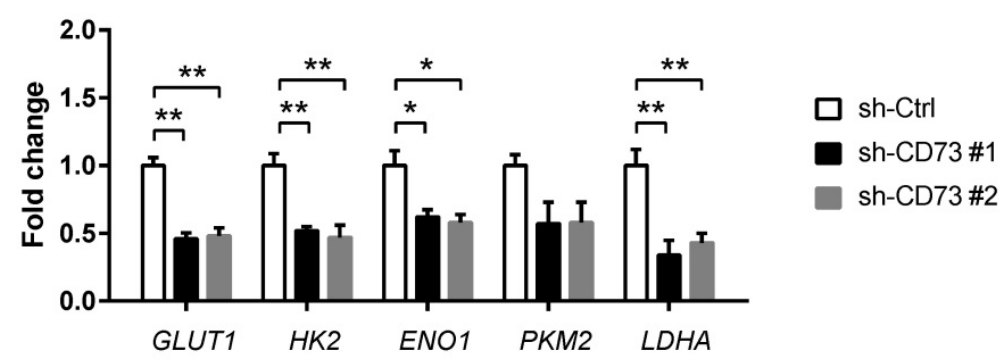

Figure 5. CD73 knockdown suppresses tumor growth in vivo. (A) A subcatenous xenograft model showed the effect of CD73 knockdown on the in vivo tumor growth of HGC-27 cells $(n=5)$. (B) Measurement of tumor weight and tumor volume in sh-Ctrl, sh-CD73 \#1 and sh-CD73 \#2 groups. (C) IHC analysis of Ki67 in sh-Ctrl, sh-CD73 \#1 and sh-CD73 \#2 tumor tissues. (D) Real-time qPCR analysis of glycolytic genes (GLUT1, HK2, ENO1, PKM2, and LDHA) in sh-Ctrl, sh-CD73 \#1 and sh-CD73 \#2 tumor tissues; GLUT1, glucose transporter type 1; HK2, hexokinase 2; ENO1, enolase 1; PKM2, pyruvate kinase M2; LDHA, lactate dehydrogenase A. *p < 0.05 ; **p < 0.01 ; ***p < 0.001 .

Emerging evidence suggests that CD73 is involved in the tumor growth and metastasis of many types of cancer, dependent on its enzyme activity in the production of adenosine in the tumor microenvironment. It has been reported that increased concentration of extracellular adenosine, which was always elevated by oxidative stress, correlated with immune and inflammation response via activation of immunosuppressive Tregs or inhibition of antitumor immune cells. CD73-mediated adenosine generation has been recognized as important purinergic signaling during cancer development and progression, which can suppress the immune response and assist tumor immune evasion in vivo via multiple signaling pathways. For example, the extracellular metabolism of ATP into adenosine through the CD38-NPP1-CD73 axis, the CD39-CD73 axis, and the NDPK/NME/NM23 axis is well-documented target system for tumor treatment [32]. CD73 could be a critical regulator that promotes tumor progression in an immune-independent manner, such as tumor stemness, epithelial-tomesenchymal transition (EMT), and therapy resistance $[32,33]$. Our data supported the concept that CD73-induced generation of adenosine promotes the Warburg effect and tumor growth in gastric cancer. Indeed, pharmacological inhibition of CD73 with APCP reduced glucose uptake and lactate release of gastric cancer cells. When tumor glycolysis was inhibited by 2-DG, APCP failed to induce an additional tumor-suppressive effect on gastric cancer cells. Acquisition of sufficient cellular buildings via the glycolysis pathway had been regarded as prerequisites for tumor growth. Here we provide a novel view of the growth-promoting roles of CD73 in gastric cancer. However, the detailed mechanism by which CD73 regulates the expression of glycolytic genes warrants further investigation. 


\section{Conclusions}

In conclusion, we report here that the hypoxic tumor microenvironment may induce CD73 expression in gastric cancer. CD73 facilitates the Warburg effect via its enzyme activity to produce adenosine. Given the important roles of the Warburg effect in facilitating tumor growth, these findings further support the pursuit of CD73 as a target for cancer therapy in gastric cancer.

\section{Abbreviations}

TCGA: The Cancer Genome Atlas; GTEx: Genotype-Tissue Expression; 2-DG: 2-deoxy-Dglucose; shRNAs: short hairpin RNAs; CM: conditioned medium; STAD: stomach adenocarcinoma; HIF1A: hypoxia-inducible factor-1alpha; GLUT1: glucose transporter type 1; HK2: hexokinase 2; ENO1: enolase 1; PKM2: pyruvate kinase M2; LDHA: lactate dehydrogenase A.

\section{Acknowledgements} study.

We thank all members for assistance with this

\section{Availability of data and material}

All data needed to evaluate the conclusions in the paper are present in the paper. Additional data related to this paper may be requested from the authors.

\section{Funding}

This work was supported by grant from Beijing Nova Programme Interdisciplinary Cooperation Project (Z191100001119140).

\section{Author Contributions}

Conception and design: MM and HJ. Analysis and interpretation of data: CXP and ZZM. Drafting of the manuscript: CXP and CY. Critical revision of the manuscript for important intellectual content: MM. Approved final version of manuscript: all authors.

\section{Competing Interests}

The authors have declared that no competing interest exists.

\section{References}

1. Allemani C, Matsuda T, Di Carlo V, Harewood R, Matz M, Niksic M, et al. Global surveillance of trends in cancer survival 2000-14 (CONCORD-3): analysis of individual records for 37513025 patients diagnosed with one of 18 cancers from 322 population-based registries in 71 countries. Lancet. 2018; 391 : 1023-75

2. Sung H, Ferlay J, Siegel RL, Laversanne M, Soerjomataram I, Jemal A, et al. Global cancer statistics 2020: GLOBOCAN estimates of incidence and mortality worldwide for 36 cancers in 185 countries. CA: a cancer journal for clinicians. 2021; 71:209-249.

3. Yoshida N, Doyama $H$, Yano $T$, Horimatsu T, Uedo N, Yamamoto $Y$, et al. Early gastric cancer detection in high-risk patients: a multicentre randomised controlled trial on the effect of second-generation narrow band imaging. Gut. 2021; 70: 67-75.

4. Cairns RA, Harris IS, Mak TW. Regulation of cancer cell metabolism. Nature reviews Cancer. 2011; 11: 85-95.

5. Doherty JR, Cleveland JL. Targeting lactate metabolism for cancer therapeutics. The Journal of clinical investigation. 2013; 123: 3685-92.

6. Hanahan D, Weinberg RA. Hallmarks of cancer: the next generation. Cell. 2011; 144: 646-74.

7. Gatenby RA, Gillies RJ. Why do cancers have high aerobic glycolysis? Nature reviews Cancer. 2004; 4: 891-9.

8. Ruzzo A, Graziano F, Bagaloni I, Di Bartolomeo M, Prisciandaro M, Aprile G, et al. Glycolytic competence in gastric adenocarcinomas negatively impacts survival outcomes of patients treated with salvage paclitaxel-ramucirumab. Gastric cancer : official journal of the International Gastric Cancer Association and the Japanese Gastric Cancer Association. 2020; 23: 1064-74.

9. Lin L, Huang H, Liao W, Ma H, Liu J, Wang L, et al. MACC1 supports human gastric cancer growth under metabolic stress by enhancing the Warburg effect. Oncogene. 2015; 34: 2700-10.

10. Liu X, Wang X, Zhang J, Lam EK, Shin VY, Cheng AS, et al. Warburg effect revisited: an epigenetic link between glycolysis and gastric carcinogenesis. Oncogene. 2010; 29: 442-50.

11. Yu H, Zhao K, Zeng H, Li Z, Chen K, Zhang Z, et al. N(6)-methyladenosine (m(6)A) methyltransferase WTAP accelerates the Warburg effect of gastric cancer through regulating HK2 stability. Biomedicine \& pharmacotherapy $=$ Biomedecine \& pharmacotherapie. 2021; 133: 111075.

12. Zhao H, Zhang L. MUC16 mutation predicts a favorable clinical outcome and correlates decreased Warburg effect in gastric cancer. Biochemical and biophysical research communications. 2018; 506: 780-6.

13. Turiello R, Pinto A, Morello S. CD73: A Promising Biomarker in Cancer Patients. Frontiers in pharmacology. 2020; 11: 609931.

14. Roh M, Wainwright DA, Wu JD, Wan Y, Zhang B. Targeting CD73 to augment cancer immunotherapy. Current opinion in pharmacology. 2020; 53: 66-76.

15. Yu M, Guo G, Huang L, Deng L, Chang CS, Achyut BR, et al. CD73 on cancer-associated fibroblasts enhanced by the A2B-mediated feedforward circuit enforces an immune checkpoint. Nature communications. 2020; 11: 515.

16. Xu Z, Gu C, Yao X, Guo W, Wang H, Lin T, et al. CD73 promotes tumor metastasis by modulating RICS/RhoA signaling and EMT in gastric cancer. Cell death \& disease. 2020; 11: 202.

17. Liu H, Li F, Zhu Y, Li T, Huang H, Lin T, et al. Whole-exome sequencing to identify somatic mutations in peritoneal metastatic gastric adenocarcinoma: A preliminary study. Oncotarget. 2016; 7: 43894-906.

18. Lu XX, Chen YT, Feng B, Mao XB, Yu B, Chu XY. Expression and clinical significance of CD73 and hypoxia-inducible factor-1alpha in gastric carcinoma. World journal of gastroenterology. 2013; 19: 1912-8.

19. Tang Z, Li C, Kang B, Gao G, Li C, Zhang Z. GEPIA: a web server for cancer and normal gene expression profiling and interactive analyses. Nucleic acids research. 2017; 45: W98-W102.

20. Zhu LL, Wu Z, Li RK, Xing X, Jiang YS, Li J, et al. Deciphering the genomic and lncRNA landscapes of aerobic glycolysis identifies potential therapeutic targets in pancreatic cancer. International journal of biological sciences. 2021; 17: 107-18.

21. Griesing S, Liao BC, Yang JC. CD73 Is Regulated by the EGFR-ERK Signaling Pathway in Non-small Cell Lung Cancer. Anticancer research. 2021; 41: 1231-42.

22. Petruk N, Tuominen S, Akerfelt M, Mattsson J, Sandholm J, Nees M, et al. CD73 facilitates EMT progression and promotes lung metastases in triple-negative breast cancer. Scientific reports. 2021; 11: 6035.

23. Wettstein MS, Buser L, Hermanns T, Roudnicky F, Eberli D, Baumeister P, et al. CD73 Predicts Favorable Prognosis in Patients with Nonmuscle-Invasive Urothelial Bladder Cancer. Disease markers. 2015; 2015: 785461.

24. Turcotte M, Spring K, Pommey S, Chouinard G, Cousineau I, George J, et al. CD73 is associated with poor prognosis in high-grade serous ovarian cancer. Cancer research. 2015; 75: 4494-503.

25. Leclerc BG, Charlebois R, Chouinard G, Allard B, Pommey S, Saad F, et al. CD73 Expression Is an Independent Prognostic Factor in Prostate Cancer. Clinical cancer research : an official journal of the American Association for Cancer Research. 2016; 22: 158-66.

26. Wu XR, He XS, Chen YF, Yuan RX, Zeng Y, Lian L, et al. High expression of CD73 as a poor prognostic biomarker in human colorectal cancer. Journal of surgical oncology. 2012; 106: 130-7.

27. Zhu J, Zeng Y, Li W, Qin H, Lei Z, Shen D, et al. CD73/NT5E is a target of miR-30a-5p and plays an important role in the pathogenesis of non-small cell lung cancer. Molecular cancer. 2017; 16: 34

28. Giatromanolaki A, Kouroupi M, Pouliliou S, Mitrakas A, Hasan F, Pappa A, et al. Ectonucleotidase CD73 and CD39 expression in non-small cell lung cancer relates to hypoxia and immunosuppressive pathways. Life sciences. 2020; 259: 118389

29. Tak E, Jung DH, Kim SH, Park GC, Jun DY, Lee J, et al. Protective role of hypoxia-inducible factor-1alpha-dependent CD39 and CD73 in fulminant acute liver failure. Toxicology and applied pharmacology. 2017; 314: 72-81.

30. Shi Y. Serine/threonine phosphatases: mechanism through structure. Cell. 2009; 139: 468-84.

31. Allard B, Pommey S, Smyth MJ, Stagg J. Targeting CD73 enhances the antitumor activity of anti-PD-1 and anti-CTLA-4 mAbs. Clinical cancer 
research : an official journal of the American Association for Cancer Research. 2013; 19: 5626-35.

32. Boison D, Yegutkin GG. Adenosine Metabolism: Emerging Concepts for Cancer Therapy. Cancer cell. 2019; 36: 582-96.

33. Yang H, Yao F, Davis PF, Tan ST, Hall SRR. CD73, Tumor Plasticity and Immune Evasion in Solid Cancers. Cancers. 2021; 13:177. 